\title{
Study on Seam Carving for Image Fingerprint
}

\author{
Makoto Fujimura*, Hideo Kuroda** and Kousuke Imamura*** \\ *Graduate School of Engineering, Nagasaki University, Nagasaki, Japan \\ **Department of Computing Fundamentals, FPT University, Hanoi, Viet Nam \\ ***Institute of Science and Engineering, Kanazawa University, Ishikawa, Japan
}

\begin{abstract}
In past several years many image contents are distributed via internet. But illegal copy problem are rise up. Multimedia fingerprint techniques are tools for resist illegal copy. However today collusion attack such as average attack is appeared. Collusion attack is efficient method for breaking copyright protection scheme of image fingerprint. This paper presented an image fingerprint scheme to resistant collusion attack which is average attack especially. Proposed image fingerprint scheme is applied seam carving technique. Experimental results show that proposed image fingerprint scheme was good performance.
\end{abstract}

Index Terms-multimedia fingerprint, seam carving, watermark, collusion attack.

\section{INTRODUCTION}

T $\mathrm{N}$ past several years image contents are published on web Ivia internet. It becomes easy to access and enjoy many multimedia contents. But problem of illegal copy of multimedia contents rise up concurrently. And some watermarking techniques are proposed for copyright protection and authentication of digital media. Digital watermarking is to embed auxiliary information into a host digital signal by imposing imperceptible signal changes. However, illegal user start to attack to watermarking of copyright protection system. Attacks on digital watermarking system are several types which are removal attacks, geometrical attack, cryptographic attack and protocol attack.

Digital image fingerprinting is an enforcing scheme for digital rights protection[1]. In digital image fingerprint scheme, unique labels are embedded into image content prior to distribution by conventional watermarking techniques. Watermarking techniques are required two types of capabilities. One is retention ability of image quality against inserting identification information into digital image content. The other one is robustness against a variety of attacks.

In recently years, multiuser collusion attacks are appeared and spread for cost-effective attacking method for attenuating each of the colluder's fingerprints. In case of traditional watermarking techniques, different identification information is embedded into each distributing image data of same content. This is reason of collusion attack, which is averaging attack especially, is possible to eliminate identification information embedded by watermarking techniques. Average attack is easy and efficiency method for eliminating embedding identification information. Average of same digital image contents can eliminate only embedding information, because different information is inserted at same location of same image content.

If distributed digital image data of same contents are different each other, average attack invoke image data quality degradation. It is possible to create different image data of same content using seam carving technique. Seam carving was proposed effective content-aware resizing of image without geometric constraints[5][6]. Seam carving process is consisted of two parts. At first, seam lines of image are searched using evaluation function of energy according to content-aware. Next, seam lines are eliminated from original image and rest of regions are connected by translation. Created resizing image by seam carving keep image quality subjectively and is different from original image in each pixels sampling location. Therefore we applied seam carving technique to digital image fingerprint for resistant average attack.

This paper propose image fingerprint scheme to resistant collusion attack which is average attack especially[3]. In this paper, we propose that new fingerprint scheme which is used seam carving techniques. Our proposed scheme is that some differently digital images embedding differently authentication information. Differently image is created invisible affine transform along image seams by seam carving techniques. Also some digital image fingerprint methods are possible to cooperate with our proposed fingerprint scheme using seam carving technique for collusion attacks resistant.

The rest of the paper is organized as follows. Section 2 describes related work. Section 3 describes the proposed image fingerprint approach in details. Section 4 shows the experimental results, and Section 4 concludes the paper.

\section{RELATED WORK}

In [1], recent some collusion-resistant fingerprinting techniques of multimedia are reviewed. There are two major 
classes of fingerprinting strategies. One is orthogonal fingerprinting and another is correlated fingerprinting.

Orthogonal fingerprinting method is based on spread-spectrum embedding techniques. This is consisted below four steps. 1) Defining carry watermark signals and transform coefficients such as discrete cosine transform (DCT). 2) Generating a watermark signal. 3) Adding the watermark to the feature signal. 4) The feature signal is embedding to original signal and obtains a watermarked signal.

Correlated fingerprinting is applied code modulation. This scheme introduces the correlation between different fingerprints in order to allow for accurate identification of the contributing fingerprints involved in a collusion attack.

\section{IMAGE FINGERPRINT SCHEME BY SEAM CARVING TECHNIQUE}

In this section, proposed image fingerprint is described in details.

\section{A. Overview of proposed image fingerprint scheme}

The image contents to transfer are created from original image. Identification information is embedded to the image by seam carving. Seam carving is image resizing technique which use not only geometric constants, but consider the image content as well. Seam carving process is consisted of two parts. One is search seam of image with energy preservation measure. The other one is resizing process to eliminate seam which is sequence of pixels. Our proposed fingerprint scheme is that some seam sequences on image are eliminated and remain regions of image are done affine transform. Figure 1 shows overview of proposed image fingerprint scheme. The transfer images are applied seam sequence respectively.

Proposed image fingerprint process is presented in below.

1. Original image is dividing to some blocks by seam lines. These blocks are bounded by seam lines. Number of blocks are determined previously and controlled by copyright management system. Figure 2 shows that divided blocks from original image. The boundary lines are seam lines.

2. Transfer image is reconstructed from divided blocks for distribution. The seam lines are eliminated from original image and transfer blocks to connect each other.



Fig. 1. Overview of image fingerprint scheme



Fig. 2. Divide blocks bounding irregular lines of seam.

\section{B. Divided blocks by seam lines}

Seam carving was proposed for effective resizing of images [5]. Seam carving supports content-aware image resizing for both reduction and expansion. A seam is an optimal 8-connected path of pixels on a single image from top to bottom, or left to right, where optimality is defined by an image energy function.

In search seam process, energy distribution of image is derived from image characteristics such like differential filter. We proposed an energy function for a variety of seam sequence. The energy function is consists of differential function and four neighborhood of pixel. ( Equation (1))

$$
\text { E_image }=\text { E_differntial }+ \text { E_4neighborbood }
$$

Figure 3 show that seam line by different evaluation function. And Figure3 (a) is seam line on image by differential energy and (b) is seam line on image by sum of differential energy and 4 neighborhood process energy. Seam line is blue color line on image in Figure2 (a) and (b). We make a comparison between Figure3 (a) and (b), these images show that each seam sequences is difference. This means that seam sequence can be controlled by energy function. In case of applied seam carving to image fingerprint, seam line of image content should be controlled for creating many types of block whereby changing the evaluation function. 


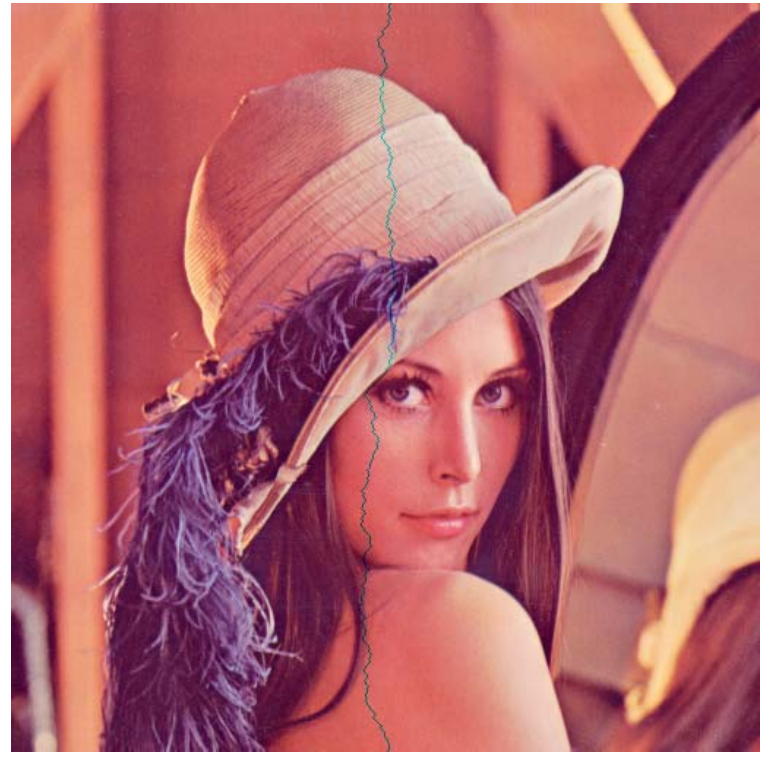

(a) Seam line(differential energy)

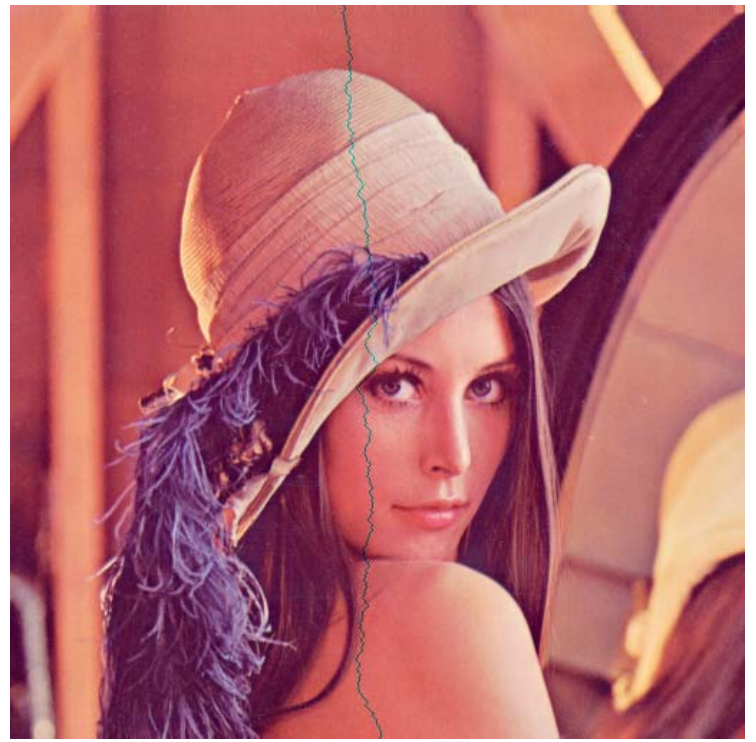

(b) Seam line(differential energy +4 neighborhood energy)

Fig. 3. Seam line by different evaluation functions.

\section{Reconstruct image with blocks of seam lines}

Original image is divided to some blocks of irregular lines in above process of dividing blocks. The seam lines are eliminated from original image and rest regions, which are bounded irregular lines, are translated in order to connect side-by-side blocks to each other.

\section{EXPERIMENTAL RESULTS}

In this section we evaluate proposed fingerprint scheme by seam carving technique. At first aim of experiment is to evaluate subjective image quality of proposed fingerprint method. And second aim of experiment is to confirm difference of sampling location of pixels between original image and reconstructed image by proposed fingerprint.

Test color images are Lenna, Sailboat, Pepper, Mandrill.
The size of original test image is $512 \times 512$. Number of seam line of rows and columns of original test image are eight of equally-divide regions. That is about $62 \times 62$ block .

At first aim, from Figure 4 to Figure 7 show the original images, the reconstructed images and seam lines, which is blue lines, draw on original images. These reconstructed image size is $504 \times 504$ because 8 lines are eliminated from rows and columns of original image. These (b)figures show that no degradation subjective image quality of proposed fingerprint scheme.

At second aim, four test image difference between original image and reconstructed image by proposed fingerprint method. Original image is cropped from pixel position (1.1), which is origin of image, to $(504,504)$ in order to match size of reconstructed image. Table 1 shows that mean absolute error(MAE) which is mean difference value between original image and reconstructed image. In Table1, range of pixel value is from 0.0 to 1.0. Figure 8 shows that absolute difference value between original image and reconstructed image. There are large difference values along shapes in images. These results mean that average attack would make degradation of image contents by proposed fingerprint.

TABLE I

MEAN ABUSOLUTE DIFFERENCE BETWEEN ORIGINAL IMAGE AND RECONSTRUCTED IMAGE

\begin{tabular}{|l|c|c|c|l|}
\hline & Lenna & Sailboat & Pepper & Mandrill \\
\hline MAE & 0.198 & 0.273 & 0.219 & 0.361 \\
\hline
\end{tabular}

\section{CONCLUSION}

We presented a fingerprint scheme using seam carving technique to resist collusion attacks. This finger print scheme is different from conventional fingerprint techniques. Experimental results showed good performance of image quality and resistant to average attack.

\section{REFERENCES}

[1] Min Wu; Trappe, W.; Wang, Z.J.; Liu, K.J.R.” Collusion-resistant fingerprinting for multimedia, "IEEE Signal Processing Magazine, March 2004,pp.15-27.

[2] H.Zhao, M.Wu, Z.Wang, and K.J.R.Liu, "Forensic Analysys of Nonlinear Collusion Attacks for Multimedia Fingerprinting", IEEE Trans. Image. Process., Vol.14, No.5,pp.646-661,2005

[3] S.He, M.Wu,"Joint Coding and Embedding Techniques for Multimedea Fingerprinting", IEEE Trans. On Information and Security, Vol.1, No.2,pp.231-247,2006

[4] W.Trappe, M.Matsushima, and S.Hirasawa, "Anti-collusion Fingerprinting for Multimedia”, IEEE Trans. Signal Process., Vol.5, No.4,pp.1069-1087,2003.

[5] Avidan, Shai and Shamir, Ariel," Seam carving for content-aware image resizing,"ACM Trans. Graph.,Vol.27 2007,

[6] Rubinstein, M.; Shamir, A. \& Avidan, S. Improved seam carving for video retargeting ACM Trans. Graph., ACM, 2008, 27, 16:1-16:9

[7] Juan Romero, Penousal Machado, Adrian Carballal and Olga Osorio, Aesthetic Classification and Sorting Based on Image Compression, WSM '09 Proceedings of the first SIGMM workshop on Social media,pp. Vol. $6625 / 2011,394-403,2011$ 




(a) Original image

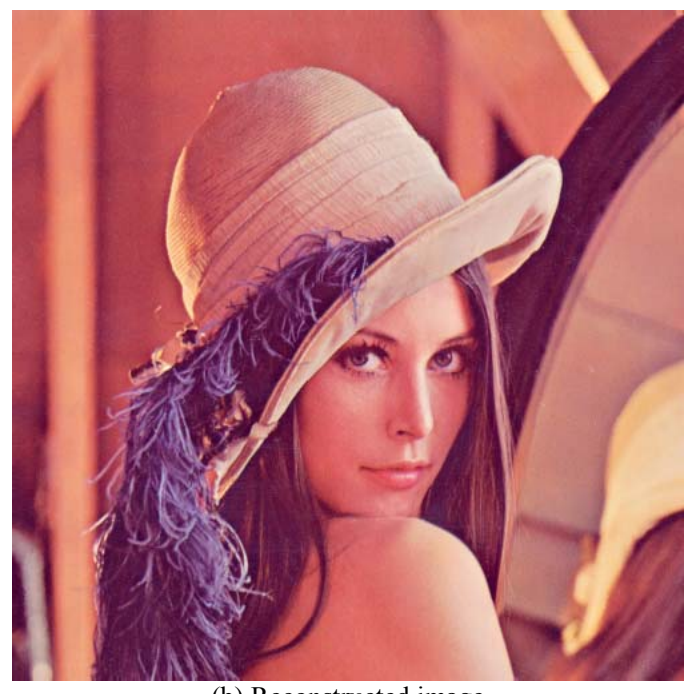

(b) Reconstructed image

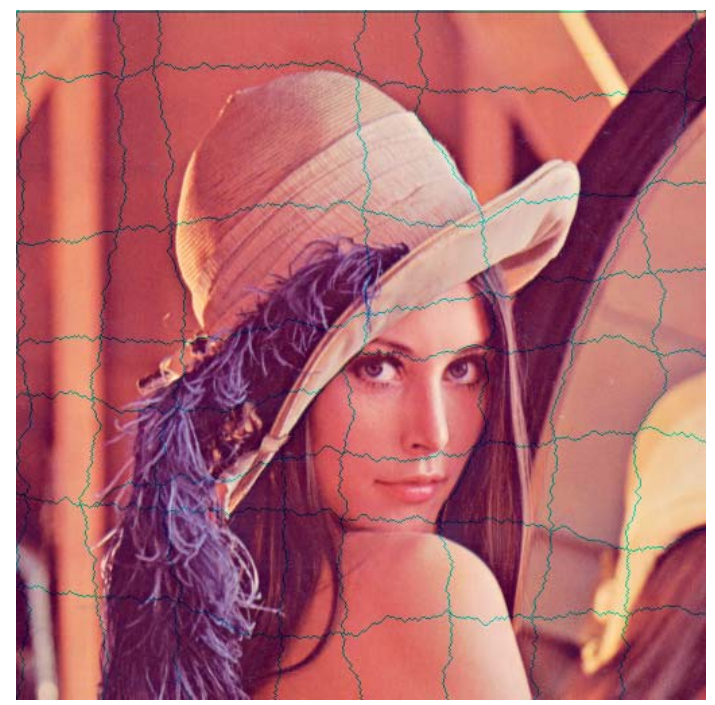

(c) Seam lines on original image

Fig. 4. Reconstructed image and seam lines on original image.(Lenna)

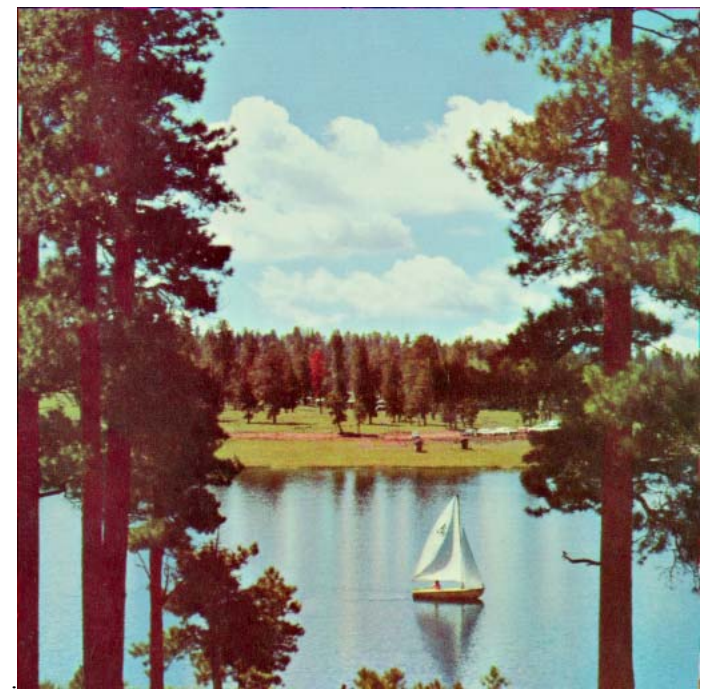

(a) Original image

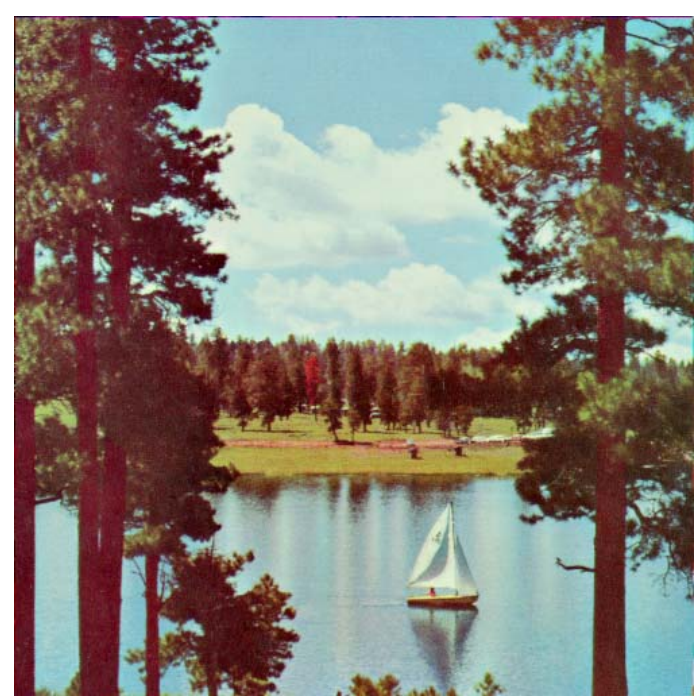

(b) Reconstructed image

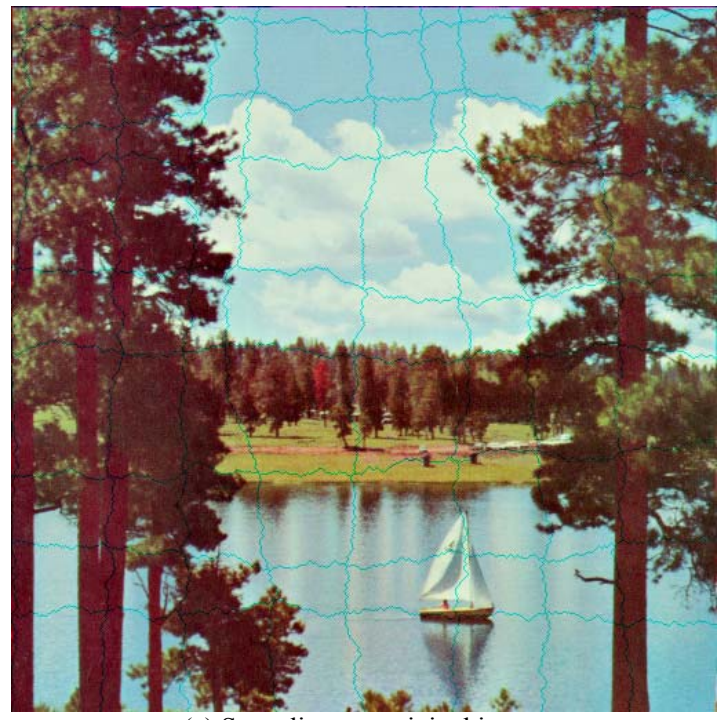

(c) Seam lines on original image

Fig. 5. Reconstructed image and seam lines on original image.(Sailboat) 


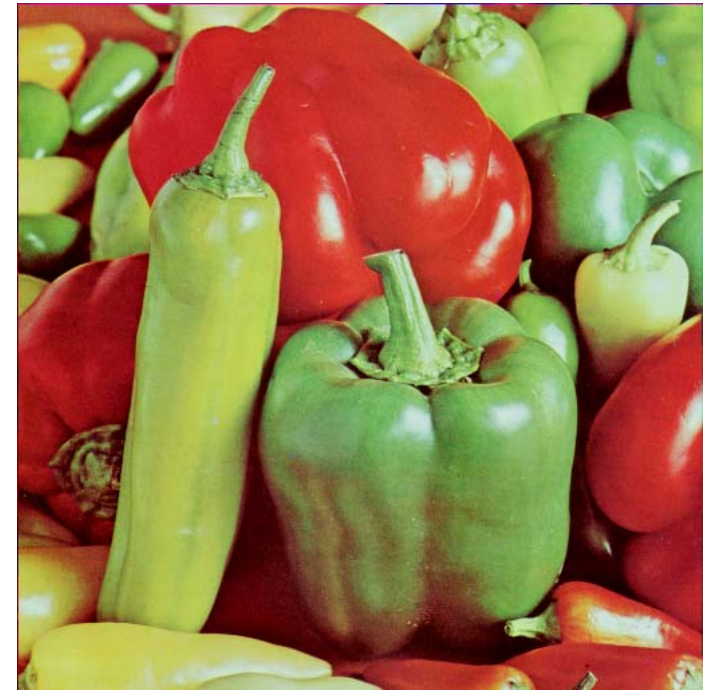

(a) Original image

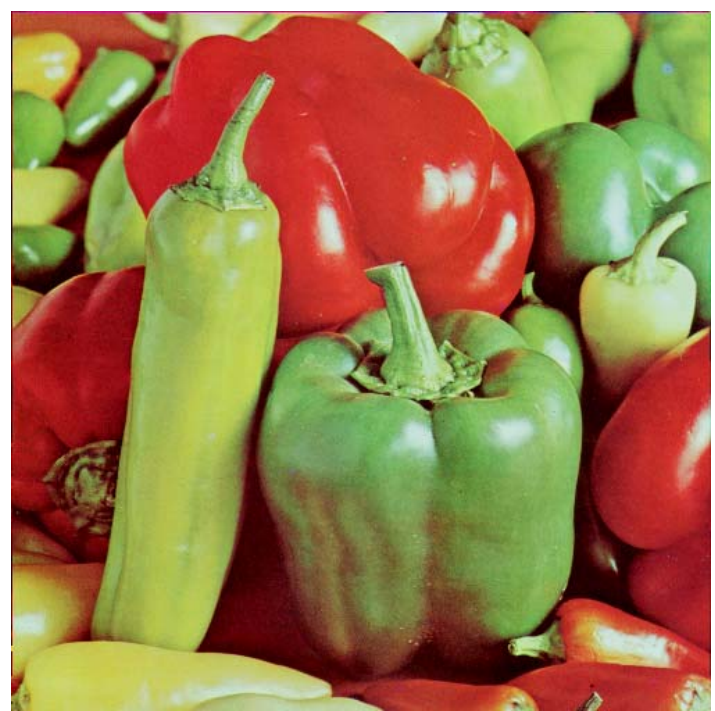

(b) Reconstructed image

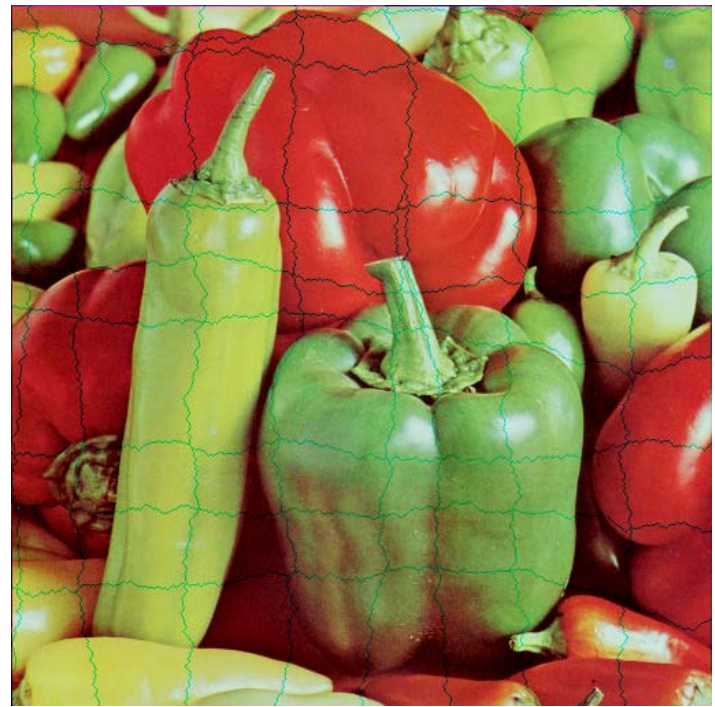

(c) Seam lines on original image

Fig. 6. Reconstructed image and seam lines on original image.(Pepper)



(a) Original image



(b) Reconstructed image

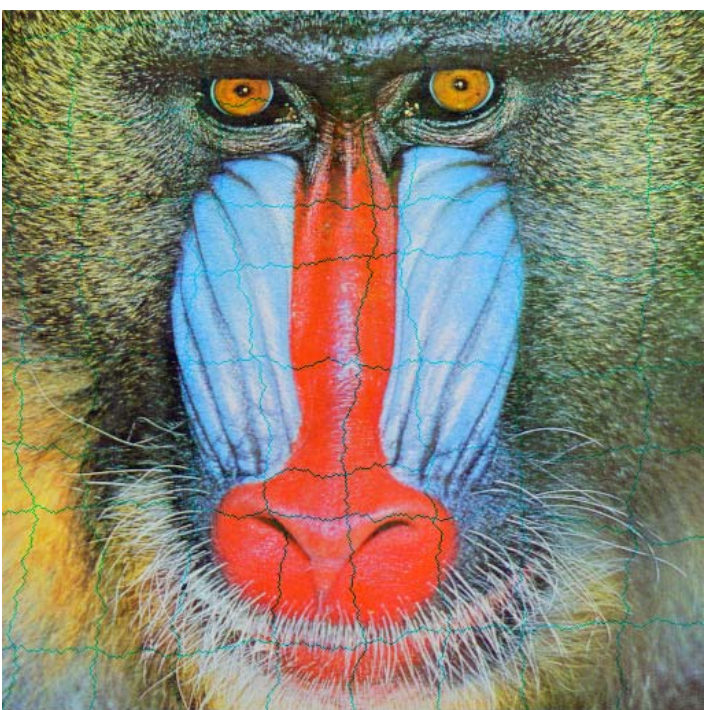

(c) Seam lines on original image

Fig. 7. Reconstructed image and seam lines on original image.(Mandrill) 


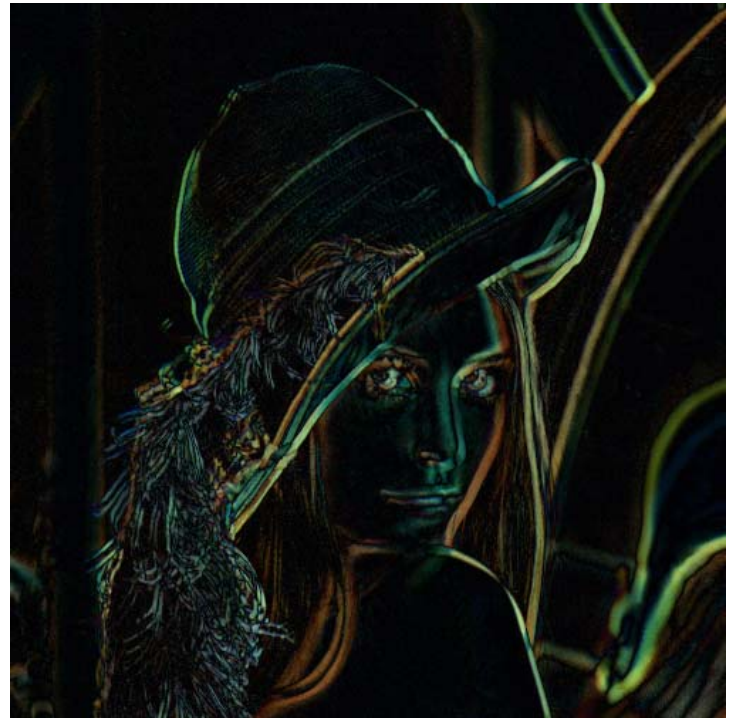

(a)Lenna

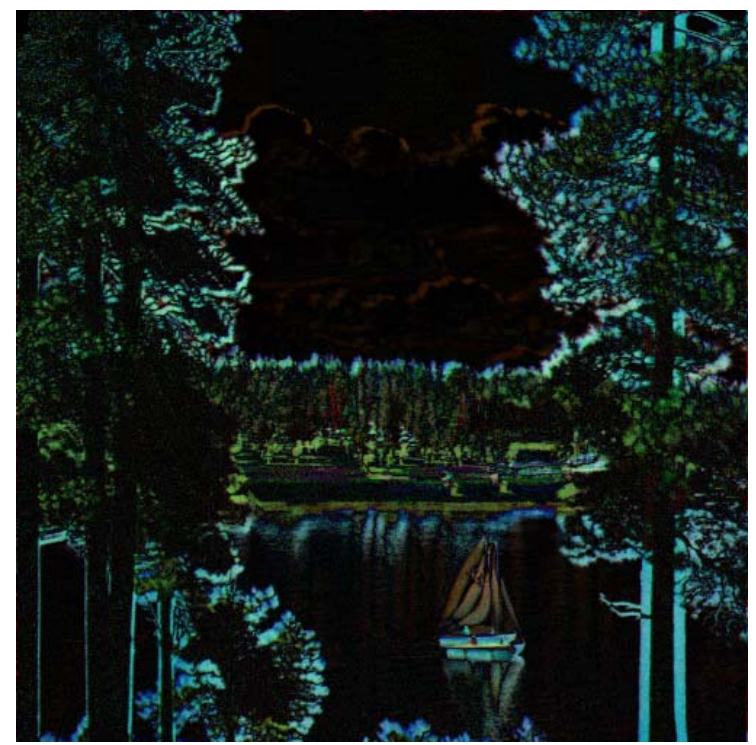

(b)Sailboat

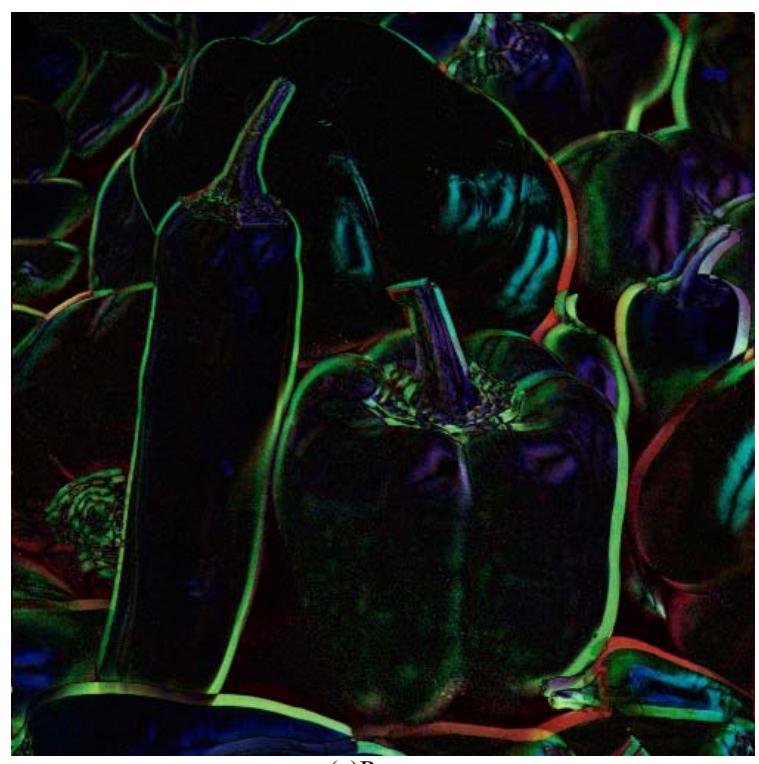

(c)Pepper

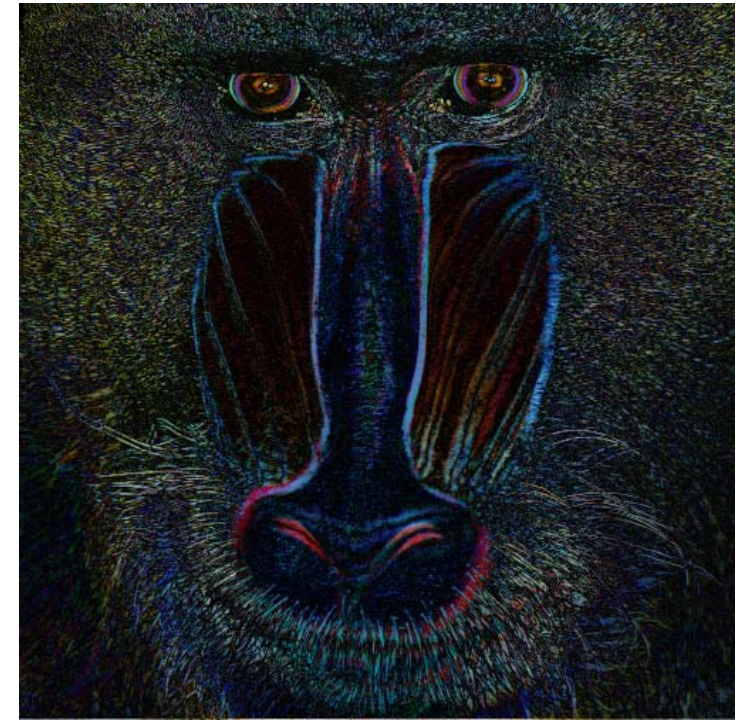

(d)Mandrill

Fig. 8. Absolute difference value between original image and reconstructed image. 